\title{
Qualidade de vida de idosos da área urbana e rural do município de Concórdia, SC
}

\section{Qulity of life of eddelyinurbanandrurd arees of themeriajplity of Concórda, SC}

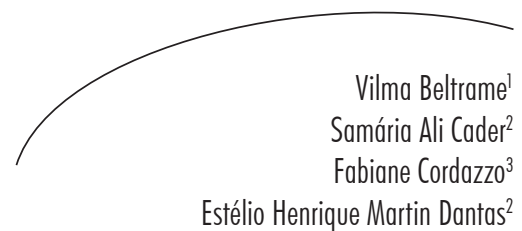

Resumo

O estudo visou a analisar a qualidade de vida (QV) entre idosos da área urbana e rural, avaliando a influência de dois fatores (zona de residência e existência ou não de patologia). Pesquisa de delineamento epidemiológico transversal, foi realizada com idosos (homens) residentes no perímetro urbano e rural do município de Concórdia, SC. A amostra consistiu de 60 idosos: grupo da área urbana (GU; $n=30$; idade $=68 \pm 8$ anos) e rural (GR; $n=30$; idade: 67 $\pm 7,88$ anos). Os dados de QV foram coletados através do formulário Medical Outcomes Study Questionnaire Health Survey (SF 36). Os resultados demonstraram que apenas as variáveis saúde $(\Delta=12,60 ; \mathrm{p}<0,0001)$ e aspectos sociais $(\Delta=28,53 ; \mathrm{p}<0,0001)$ obtiveram diferença significativa $(\mathrm{p}<0,05)$ intergrupos, sendo os valores do GR superiores (mais favoráveis) aos do GU. O teste do qui-quadrado revelou diferença significativa $\left(\chi^{2}: 4,34 ; p=0,037\right)$ intergrupos, sendo o GR o grupo que possuiu menor quantitativo de indivíduos com patologia. Desta forma infere-se que, na amostra analisada, o GR obteve melhora na qualidade de vida, em relação à saúde e aos aspectos sociais, quando comparados ao GU.

\section{Abstract}

The study aimed to analyze the quality of life (QOL) among elderly people in urban and rural areas, assessing the influence of two factors (area of residence and presence or absence of pathology). The research design of epidemiological cross-sectional was conducted with elderly people (men) living in the urban and rural municipality of Concordia, SC. The sample consisted of 60 elderly people: group of the urban area (UG, $\mathrm{n}=30$, age $=68 \pm 8$ years) and rural ( $\mathrm{RG}, \mathrm{n}=30$, age: $67 \pm 7.88$ years). QOL data were collected through the Medical Outcomes Study Questionnaire Form Health

Palavras-chave: Idoso. População Urbana. População Rural. Qualidade de Vida. Concórdia, SC.
Key words: Aged. Urban Population. Rural Population. Quality of Life. Concordia City.

\footnotetext{
Programa de Pós-Graduação em Biociências na Saúde. Universidade do Oeste do Estado de Santa Catarina. Joaçaba, SC, Brasil.

2 Laboratório de Biociências da Motricidade Humana. Universidade Federal do Estado do Rio de Janeiro. Rio de Janeiro, RJ, Brasil.

3 Curso de Enfermagem. Universidade do Contestado. Concórdia, SC, Brasil.
} 
Survey (SF 36). The results showed that only health $(\Delta=12.60, \mathrm{p}<0.0001)$ and social $(\Delta=28.53, \mathrm{p}<0.0001)$ variables had significant difference $(\mathrm{p}<0.05)$ between groups and the values of RG higher (more favorable) to the UG. The chi-square revealed a significant difference $\left(\chi^{2}: 4.34, \mathrm{p}=0.037\right)$ between groups, with the RG group that has a smaller quantity of individuals with disease. Thus, it is inferred that in our sample, the RG obtained improvement in quality of life in relation to health and social aspects when compared to the UG.

\section{INTRODUÇÃO}

A população idosa cresce rapidamente e, assim, cresce a preocupação com o bem-estar do idoso em todos os aspectos, inclusive no da qualidade de vida, ${ }^{1}$ sendo necessário estudos específicos para esta faixa etária. ${ }^{2}$

Existem vários protocolos para avaliar a qualidade de vida (QV), ${ }^{3-5}$ a qual pode ser definida como um bem-estar subjetivo que reflete a distância entre as expectativas e esperanças de um indivíduo e sua experiência efetiva. A adaptação humana favorece o ajuste das esperanças à realidade concreta que se vive, de forma a se satisfazer com o que aparenta ser a possibilidade concreta. Isto facilita a manutenção de uma razoável QV, mesmo diante de circunstâncias difíceis. ${ }^{6}$ Reconhecendo a subjetividade de QV como medida crucial para descrever o bem-estar subjetivo, há que se observar que esta subjetividade pode depender da satisfação da comunhão de condições ambientais, econômicas, sociais, físicas, emocionais, psíquicas e mentais. ${ }^{7}$

Todavia, a grande variação nas taxas de envelhecimento populacional, nas diferentes regiões brasileiras, faz com que a transposição das disposições dos direitos do idoso para o cotidiano da população seja um dos grandes desafios para os próximos anos e dependa do contexto regional no qual ele se insere. ${ }^{8}$ Neste sentido, alguns estudos têm-se dedicado a estudar a senescência sob novas óticas, sendo poucas as pesquisas que abordam o tema do envelhecimento no ambiente rural. ${ }^{9}$ Esse ambiente é caracterizado por municípios que possuem menos de 25.000 habitantes, e 75\% dos municípios brasileiros se enquadram nesta definição, onde residem cerca de 40 milhões de pessoas. Estes municípios rurais são constituídos por um núcleo urbano, que corresponde à sede municipal, e um núcleo agrário, onde se desenvolvem atividades de agricultura e pecuária. ${ }^{10}$

Dentro deste contexto, o Short-Form 36 (SF36), é utilizado para avaliar a qualidade de vida na maioria desses estudos. ${ }^{11,12} \mathrm{E}$ interessante ressaltar a escolha do SF-36 em nosso estudo para obter maior gama de informações, devido à simplicidade de sua aplicação para a população selecionada. Neste sentido, o estudo teve como objetivo avaliar e comparar os níveis de qualidade de vida de idosos residentes na área urbana e na rural do município de Concórdia, Estado de Santa Catarina, identificando a influência de dois fatores: local de residência e existência ou não de patologia.

\section{MATERIAIS E MÉTODOS}

Amostra

Trata-se de estudo epidemiológico e transversal, realizado com idosos do sexo masculino residentes no perímetro urbano e rural do município de Concórdia, SC, que frequentavam grupos de idosos de Barra do Tigre, localizado no perímetro rural e do grupo dos idosos do grupo do bairro Nazaré e do bairro da Gruta, no período urbano.

O estudo foi aprovado pelo Comitê de Ética em Pesquisas da UnC-Concórdia (protocolo $n^{\circ}$ 272/07). Foram explicados os objetivos, riscos e benefícios da pesquisa, garantindo-se o sigilo e a confidencialidade das informações individuais 
e que só dados globais seriam divulgados à comunidade acadêmica, conforme as normas para a realização de pesquisas envolvendo seres humanos, atendendo aos critérios da Declaração de Helsinki ${ }^{13}$ e as recomendações da Resolução no 196/96 do Conselho Nacional de Saúde. ${ }^{14}$ Todos os sujeitos concordaram em participar da pesquisa mediante assinatura do termo de consentimento livre e esclarecido, que versa sobre as exigências éticas para a realização de pesquisas com seres humanos.

A seleção da amostra foi depurada, obedecendo aos critérios de inclusão e exclusão. Como critério de inclusão, os indivíduos da amostra deveriam participar do grupo de idosos há pelo menos seis meses, ser do sexo masculino, ter idade igual ou superior a 60 anos e aceitar assinar o termo de consentimento livre e esclarecido de participação em pesquisa. Foi considerado critério de exclusão os idosos que fizessem uso de medicamentos que pudessem causar distúrbios da atenção, o que poderia mascarar alguns resultados do questionário.

Após o crivo dos critérios de inclusão e exclusão (através de um questionário de anamnese), a amostra contou com um quantitativo de 82 idosos, que foram convidados a participar da avaliação do questionário de qualidade de vida no dia seguinte. Entretanto, devido à desistência ( $\mathrm{n}=22$ idosos) na participação do questionário, o estudo findou com um quantitativo de 60 idosos: grupo da área urbana (GU; $\mathrm{n}=30$; idade $=68 \pm 8$ anos) e rural (GR; $\mathrm{n}=30$; idade: $67 \pm 7,88$ anos).

\section{Procedimentos}

Os dados foram coletados através do formulário Medical Outcomes Study Questionnaire Health Survey (SF 36), ${ }^{15}$ traduzido para o português, instrumento genérico de avaliação da qualidade de vida composto por 36 itens que avaliam as seguintes dimensões: 1) Capacidade funcional: desempenho das atividades diárias, como capacidade de cuidar de si, vestir-se, tomar banho e subir escadas; 2) Aspectos físicos: impacto da saúde física no desempenho das atividades diárias e ou profissionais; 3) Dor: nível de dor e o impacto no desempenho das atividades diárias e ou profissionais; 4) Estado geral de saúde: percepção subjetiva do estado geral de saúde; 5) Vitalidade; 6) Aspectos sociais: reflexo da condição de saúde física nas atividades sociais; 7) Aspectos emocionais: reflexo das condições emocionais no desempenho das atividades diárias e ou profissionais; 8) Saúde mental: escala de humor e bem-estar.

Apresenta o escore final de zero a cem, no qual zero corresponde ao pior escore geral de qualidade de vida e cem ao melhor escore geral de qualidade de vida. É chamado de raw scale, por que o valor final não apresenta nenhuma unidade de medida.

\section{Tratamento estatístico}

Os dados da estatística descritiva foram apresentados como média, desvio-padrão, mediana, erro-padrão, mínimo, máximo e delta absoluto $(\Delta)$. A normalidade dos dados foi analisada através dos testes de Shapiro-Wilk. Para a comparação intergrupo foi utilizado o teste de Mann-Whitney (escala ordinal). Para a análise da associação entre as variáveis (grupo e existência de patologia), foi utilizado o tese do qui-quadrado $\left(\chi^{2}\right)$. $\mathrm{Na}$ área de qualidade de vida, é importante abordagem para determinar se as diferenças observadas são clinicamente relevantes, com o cálculo do tamanho do efeito, uma das estimativas utilizadas. ${ }^{16,17}$ A magnitude dos efeitos das diferenças nos escores global e de domínio entre os dois grupos de interesse foi calculado dividindo-se as diferenças de valores médios entre o desvio padrão (SD) do grupo de comparação. Seguiu-se a abordagem de Cohen para a interpretação desses efeitos: 0,20 representa um pequeno efeito, 0,50 um efeito moderado e maior efeito $\geq 0,80 .{ }^{18}$ Também foi realizado o cálculo do poder do experimento. O programa SPSS 18.0 foi utilizado para a análise dos dados e considerou-se nível de 5\% de significância. 


\section{RESULTADOS}

A tabela 1 traz a análise descritiva e de normalidade dos moradores da área urbana. Nela se pode observar que apenas as variáveis capacidade funcional e aspectos sociais obtiveram distribuição normal dos dados.

Tabela 1 - Análise descritiva e inferencial de Shapiro-Wilk dos indivíduos moradores na área urbana. Concórdia, SC, 2008.

\begin{tabular}{lcccccccc}
\hline & $\begin{array}{c}\text { Capacidade } \\
\text { Funcional }\end{array}$ & $\begin{array}{c}\text { Aspectos } \\
\text { Físicos }\end{array}$ & Dor & Saúde & Vitalidade & $\begin{array}{c}\text { Aspectos } \\
\text { Sociais }\end{array}$ & $\begin{array}{c}\text { Aspecto } \\
\text { Emocional }\end{array}$ & $\begin{array}{c}\text { Saúde } \\
\text { Mental }\end{array}$ \\
\hline Média & 70,33 & 51,67 & 78,60 & 62,20 & 62,67 & 50,00 & 60,03 & 77,07 \\
Erro padrão & 3,84 & 6,77 & 3,27 & 1,55 & 1,85 & 0,00 & 7,40 & 2,34 \\
Mediana & 75,00 & 50,00 & 84,00 & 62,00 & 60,00 & 50,00 & 67,00 & 80,00 \\
Desvio padrão & 21,01 & 37,10 & 17,90 & 8,46 & 10,15 & 0,00 & 40,54 & 12,82 \\
Mínimo & 15,00 & 0,00 & 31,00 & 35,00 & 45,00 & 50,00 & 0,00 & 36,00 \\
Máximo & 100,00 & 100,00 & 100,00 & 80,00 & 95,00 & 50,00 & 100,00 & 100,00 \\
SW & 0,170 & 0,002 & 0,018 & 0,002 & 0,000 & 1,000 & 0,000 & 0,018 \\
\hline
\end{tabular}

SW: p-valor do teste de Shapiro Wilk.

A análise descritiva e a normalidade dos grupos de moradores da zona rural estão expostas na tabela 2. Através do teste de
Shapiro-Wilk pode ser verificado que apenas a variável saúde obteve uma distribuição normal dos dados.

Tabela 2 - Análise descritiva e inferencial de Shapiro-Wilk dos indivíduos moradores na área rural. Concórdia, SC, 2008.

\begin{tabular}{lcccccccc}
\hline & $\begin{array}{c}\text { Capacidade } \\
\text { Funcional }\end{array}$ & $\begin{array}{c}\text { Aspectos } \\
\text { Físicos }\end{array}$ & Dor & Saúde & Vitalidade & $\begin{array}{c}\text { Aspectos } \\
\text { Sociais }\end{array}$ & $\begin{array}{c}\text { Aspecto } \\
\text { Emocional }\end{array}$ & $\begin{array}{c}\text { Saúde } \\
\text { Mental }\end{array}$ \\
\hline Média & 79,00 & 55,83 & 81,43 & 74,80 & 63,67 & 78,53 & 62,23 & 78,67 \\
Erro padrão & 4,47 & 6,85 & 4,84 & 2,80 & 2,22 & 4,00 & 7,29 & 3,34 \\
Mediana & 90,00 & 75,00 & 100,00 & 72,00 & 65,00 & 88,00 & 67,00 & 80,00 \\
Desvio padrão & 24,47 & 37,53 & 26,51 & 15,33 & 12,17 & 21,94 & 39,91 & 18,30 \\
Mínimo & 5,00 & 0,00 & 10,00 & 35,00 & 30,00 & 25,00 & 0,00 & 24,00 \\
Máximo & 100,00 & 100,00 & 100,00 & 100,00 & 80,00 & 100,00 & 100,00 & 100,00 \\
SW & 0,000 & 0,001 & 0,000 & 0,295 & 0,000 & 0,001 & 0,000 & 0,000 \\
\hline
\end{tabular}

SW: p-valor do teste de Shapiro Wilk. 
A figura 1 apresenta a análise comparativa da qualidade de vida entre os dois grupos (GU x GR). Pelo fato de o questionário ter característica de uma escala ordinal, o teste utilizado foi o nãoparamétrico de Mann-Whitney. Através dele pode- se observar que apenas as variáveis saúde (IC,95\%: $-19,00 /-6,20 ; \mathrm{p}<0,001)$ e aspectos sociais (IC, $95 \%$ : $-36,55 /-20,52 ; \mathrm{p}<0,001)$ obtiveram diferença significativa intergrupos, sendo os valores do GR superiores (mais favoráveis) aos do GU.

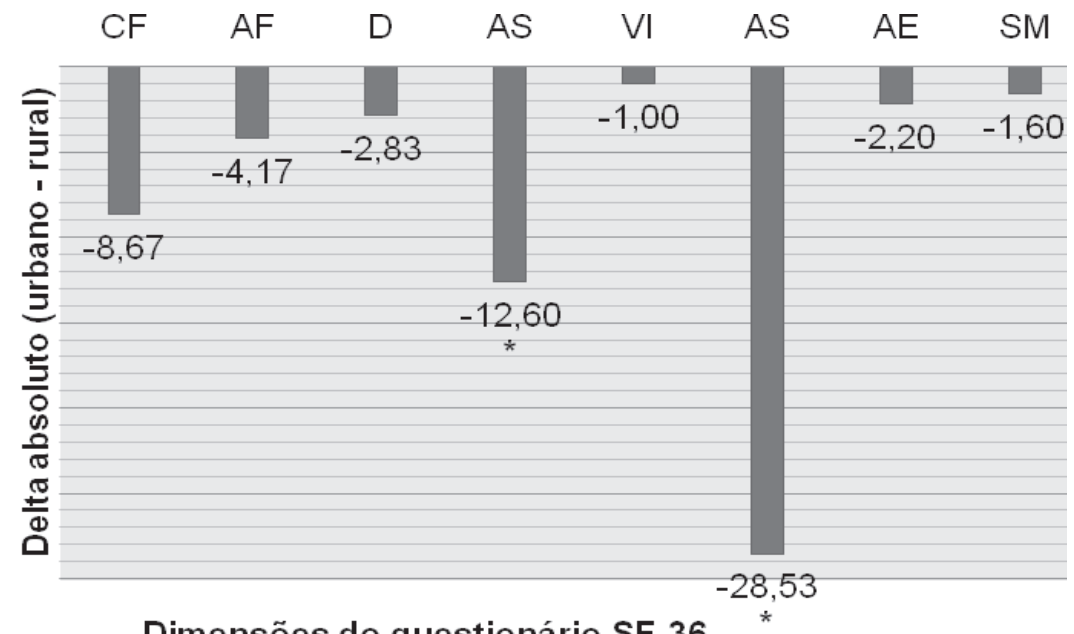

Dimensões do questionário SF-36

${ }^{*} \mathrm{p}<0,05$; a favor do grupo rural

CF: capacidade funcional; AF: aspectos físicos; D: dor; AS: saúde; VI: vitalidade; AS: aspectos sociais; AE: aspecto emocional; SM: saúde mental

Figura 1 - Análise comparativa da qualidade de vida intergrupos

Embora tenha sido realizada a comparação intergrupos sobre a qualidade de vida, foi observado que, como uma das diferenças significativas foi em relação à saúde, optou-se por realizar o teste de qui-quadrado (figura 2), separando os grupos em subgrupos com ou sem presença de comorbidades.

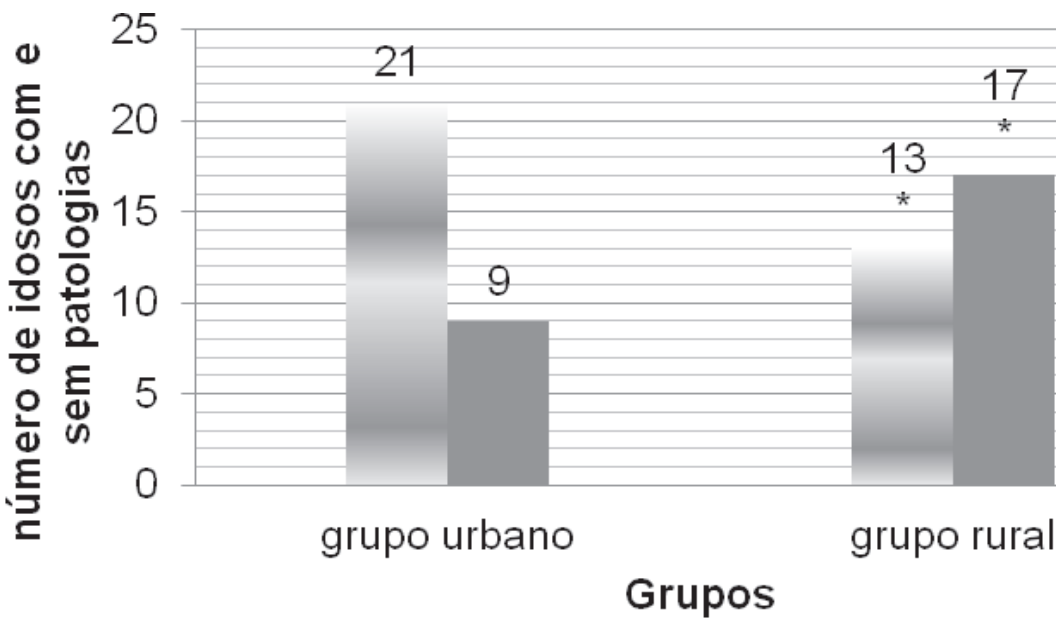

= possui patologia não possui patologia

${ }^{*} \mathrm{p}<0,05$; grupo urbano x grupo rural

Figura 2 - Separação dos grupos daqueles que possuem ou não patologias 
Através dos dados apresentados na figura 2, o teste do qui-quadrado $(2: 4,34)$ revelou uma diferença significativa $(p=0,037)$ intergrupos, sendo o GR o grupo que possuiu um menor quantitativo de indivíduos com patologia. Em relação ao tamanho do efeito das duas variáveis estatisticamente diferentes intergrupos, segundo o índice de Cohen, encontrou-se grande efeito da saúde $(1,48)$ e do aspecto social $(2,85)$ da referida amostra. $\mathrm{O}$ poder do experimento, relacionado às duas variáveis significativas, foi de $97 \%$ no aspecto geral de saúde e, no aspecto social, de $99 \%$.

\section{DISCUSSÃO}

Existem vários protocolos que avaliam a qualidade de vida. ${ }^{3-5,18-20}$ No entanto, o SF-36 é usado na avaliação desta variável na maior parte dos estudos. Assim, a discussão é norteada com a comparação da aplicação do SF-36, bem como de outros questionários, com populações de diversos países.

Embora o presente estudo tenha apresentado boa qualidade de vida nos moradores da zona rural, os resultados do trabalho de Cleary e Howell ${ }^{11}$ vêm de encontro a esses achados. Nessa pesquisa, que teve por objetivo determinar a percepção de qualidade de vida relacionada com a saúde (QVRS), de idosos (homens e mulheres) da área rural de Idaho, ao sudeste dos Estados Unidos da América (EUA), contou-se com a participação de 95 indivíduos. O questionário utilizado foi o SF-36 versão 2. Os escores da amostra foram comparados com os estabelecidos normativos para a população geral adulta dos EUA e especificamente para os valores normativos para as pessoas idosas. Em geral, a QVRS dos participantes foi menor que a da população geral. No entanto, as mulheres e homens com idades $\geq 75$ anos apresentaram maiores escores relacionados ao componente físico sumário (CFS), quando relacionados aos valores normativos de sua idade e sexo compatíveis.

Os principais objetivos de um artigo, realizado com 1.680 idosos (selecionados de forma aleatória), na província de Anhui, China, foram analisar as distribuições e correlação entre as condições do sono e da qualidade de vida de moradores nas áreas rurais. ${ }^{12}$ Estas foram avaliadas de forma independente, utilizando o índice de qualidade do sono de Pittsburgh (IQSP) e a escala SF-36, com a mesma versão utilizada na vigente investigação. A pontuação média do PSQI foi 7,43 \pm 4.13 . As classificações do sono da amostra foram: boa (29,4\%), comum $(24,3 \%)$ ou pobre $(46,3 \%)$. Sexo e qualidade do sono foram diferentes em termos de qualidade de vida entre todos os fatores $(\mathrm{P}<0,05)$. Os homens apresentaram melhor qualidade que as mulheres, e os de classificação pobre de sono apresentaram pior qualidade de vida, em comparação com aquela com melhores condições de sono. Fatores como estar casado / viver sozinho / economicamente independente teve melhor função social, muitas vezes comer carne ou trigo foram significativos preditores para ter condições de um bom sono. No entanto, fatores decorrentes uma melhor educação recebida; o próprio viver só, mesmo com uma vitalidade insuficiente, apesar de não comprometer a saúde em geral, eram os observados nos sujeitos que possuíam doenças crônicas (lombalgia, coronariopatia ou problemas estomacais), e naqueles que apresentavam uma ingestão menor de carne e arroz. Estes, também, foram os principais preditores de uma pior condição de qualidade de sono, dados estes que corroboram com os achados de Yousefian. ${ }^{21}$

Desta forma, os dados mostraram que a qualidade do sono entre os idosos rurais na província de Anhui não era satisfatória, recomendando-se melhores condições de saúde, gerais e nutrição para ser melhorada a qualidade do sono, a qual interferirá na qualidade de vida. Embora esses dados se contrapõnham àqueles expostos na tabela 3 e 4 , pode-se encontrar um viés como limitação deste estudo, a falta de controle nutricional com vistas a uma análise comparativa mais apurada com o trabalho de Li et al. ${ }^{12}$

Em estudo realizado no Peru, ${ }^{22}$ encontramse dados que se assemelham aos resultados do presente estudo. Seu objetivo foi explorar 
se havia diferença na percepção autorreferida da qualidade de vida entre os imigrantes rural-urbanos e grupos urbanos, utilizando o questionário WHOQOL-brief. Um total de 307 indivíduos (imigrantes: $\mathrm{n}=19$ e não imigrantes: $\mathrm{n}=116$ ) foram pesquisados. Comparados com o grupo urbano, os imigrantes relataram menor qualidade de vida tanto no escore global, assim como na saúde psicológica e os domínios do ambiente em que vivem atualmente, relatando melhora apenas no domínio saúde física. Neste sentido, o impacto da migração rural-urbana na qualidade de vida sugere um efeito diferencial, e por isso possui a mesma tendência deste trabalho, uma vez que se relatou piora nos domínios saúde psicológica e ambiente, isto, em última análise, significa que no ambiente rural deveriam se sentir melhores.

Um amplo estudo realizado com três populações de idosos urbanos, rurais e de remotas ilhas (Shihpai, Yuli, e Kinmen, respectivamente, em Taiwan), teve por objetivo estudo fornecer as normas para a medida do estado de saúde através do questionário SF-36 e explorar a relação entre dados demográficos ou fatores característicos ou SF-36. ${ }^{23}$ Um total de 6.503 indivíduos que tinham pelo menos 65 anos de idade foram convidados, e ao final do estudo foram contabilizados $4.424(73,1 \%)$ participantes. Os resultados demonstraram que as normas urbanas foram significativamente maiores do que as normas rurais em sete escalas do SF-36, e significativamente maiores do que as normas ilha remota em quatro escalas, incluindo o funcionamento físico (84,6 vs 77,1), limitações devido a problemas físicos $(77,8$ vs 70,2$)$, estado geral de saúde $(70,5$ vs 65,5$)$ e limitações de papéis por problemas emocionais $(90,6$ vs 85,2$)$. As normas das ilhas remotas foram significativamente maiores $(p<0,05)$ do que as normas urbanas e rurais de vitalidade e mental nas escalas da saúde. Nas análises multivariadas, as mulheres apresentaram escores significativamente mais pobres em todas as escalas do SF-36 que os homens ( $p<0,05)$, exceto para o social em funcionamento. Dezenas de escalas foram reduzidas significativamente de acordo com o número de doenças crônicas, que, neste último aspecto, possui a mesma tendência encontrada nesta pesquisa, a qual revelou que o GR, além de ter signficativa melhora do specto saúde, também apresentou menor número de patologias. Houve diferenças significativas entre as áreas urbanas, rurais e remotas populações insulares de idosos na maioria das escalas do SF36. Os idosos urbanos tiveram a maior qualidade de saúde da vida, na maioria das escalas, particularmente sobre as escalas de saúde física. A população de idsosos da ilha remota teve a maior pontuação na vitalidade e escalas de saúde mental, enquanto a população idosa rural teve a pior qualidade de vida relacionada à saúde, principalmente as mulheres.

Dados semelhantes a esse trabalho foram observados por Pereira et. al., ${ }^{24}$ que verificaram as características biofísicas de mulheres idosas na Barra da Tijuca, região de classe média alta do Rio de Janeiro. Em relação à qualidade de vida geral (QVG), o instrumento usado no estudo foi o WHOQOL-100, onde o escore de QVG foi $15,57 \pm 1,40$. Alencar et. al., ${ }^{25}$ utilizando o questionário WHOQOL-old, já distanciaram seus resultados de todos os demais abordados bem como do atual artigo, pois não encontraram diferença significativa na qualidade de vida nas diferentes regiões demográficas. Da mesma forma, o estudo de Alencar et al., ${ }^{26}$ realizado no Brasil (Estado do Ceará), em relação a esse último trabalho, observou na análise dos resultados que as idosas foram classificadas com média do QVG-old (do questionário WHOQOL-old) igual ou superior a 13 como sendo detentoras de qualidade de vida suficiente; $46 \%$ das idosas residentes na área urbana apresentaram níveis iguais ou acima de 13, sobrepondo-se aos $40 \%$ dos indivíduos residentes na zona rural, que obtiveram níveis superiores a 13 , porém sem diferenças estatísticas.

Deve-se ressaltar que, dentre as limitações enfrentadas por este estudo, pode ser citada a dificuldade em encontrar estudos de qualidade, no Brasil, que tenham realizado a comparação da qualidade de vida entre os ambientes urbano e rural. Os poucos trabalhos publicados estão referenciados no presente estudo. 


\section{CONCLUSÃO}

Observa-se, após a análise dos dados e da discussão da literatura, que o fato de residir em perímetros urbanos acarreta, em alguns domínios, melhores escores. Isso possivelmente ocorre pelo fato de os residentes em meio urbano possuírem melhores níveis econômicos, o que facilitaria a acessibilidade aos meios de comunicação, saúde, lazer e aquisição de bens de consumo, dentre outros.

No entanto, o presente estudo observou que em relação aos aspectos sociais e de saúde o GR teve resultados mais satisfatórios, apresentando ainda menor número de indivíduos com

\section{REFERÊNCIAS}

1. Prado SD. A Revista Brasileira de Geriatria e Gerontologia e os novos horizontes da pesquisa brasileira no cenário internacional. Revista brasileira de geriatria e gerontologia. 2009; 12(1): 5 .

2. Dias Júnior CS, Costa CS, Lacerda MA. O envelhecimento da população brasileira: uma análise de conteúdo das páginas da REBP. Revista brasileira de geriatria e gerontologia. 2006; 9(2): 7-24.

3. Hawthorne G, Herrman H, Murphy B. Interpreting the WHOQOL-Brèf: preliminary population norms and effect sizes. Soc Indic Res 2006; 77(1): 37-59.

4. WHOQOL. Versão em português dos instrumentos de avaliação de Qualidade de Vida (WHOQOL); 1998. Disponível em: http://www.ufrj.br/psiq/ whoqol.html.

5. Fleck MP, Chachamovich E, Trentini C. Development and validation of the Portuguese version of the WHOQOL-OLD module. Rev Saude Publica. 2006; 40 (5): 785-91.

6. Zhang J, Huang H, Ye M, Zeng H. Factors influencing the subjective well being (SWB) in a sample of older adults in an economically depressed area of China. Arch Gerontol Geriatr. 2008; 46(3): 335-47.

7. Veras R. Em busca de uma assistência adequada à saúde do idoso: revisão de literatura e aplicação de um instrumento de detecção precoce e de previsibilidade de agravos. Cad Saúde Pública 2003; 19(3): 705-15. patologias. Todavia, alguns parâmetros, como estado nutricional, nível de estresse e nível socioeconômico, não foram controlados para se concluir por que os achados desta investigação se contrapõem aos da maioria dos demais estudos em discussão, embora esses também não tenham controlado tais variáveis, as quais podem influenciar os resultados.

Neste sentido, sugere-se que novos estudos transversais, porém multicêntricos, sejam realizados com uma avaliação mais completa, a fim de contemplar as variáveis intervenientes não controladas nos estudos realizados até o presente momento.

8. Alencar NA, Aragão JCB, Ferreira MA, Dantas EHM. Avaliação da qualidade de vida em idosas residentes em ambientes urbano e rural. Revista brasileira de geriatria e gerontologia. 2010; 13 (1): 103-9.

9. Martins CR, Albuquerque FJB, Gouveia CNNA, Rodrigues CFF, Neves MTS. Avaliação da qualidade de vida subjetiva dos idosos: uma comparação entre os residentes em cidades rurais e urbanas. Estudos Interdisciplinares sobre o Envelhecimento 2007; 11: 135-54.

10. McHorney CA, Ware JE, Raczek AE. The MOS 36-Item Short-Form Health Survey (SF-36): II. Psychometric and Clinical Tests of Validity in Measuring Physical and Mental Health Constructs. Med Care 1993; 31(3): 247-63.

11. Cleary KK, Howell DM. Using the SF-36 to determine perceived health-related quality of life in rural Idaho seniors. J Allied Health. 2006; 35(3):156-61.

12. Li J, Dong Q, Liu JJ, Dong YH, Yang LS, Ye DQ, et al. Sleep and quality of life among rural elderly in Anhui province. Zhonghua Liu Xing Bing Xue Za Zhi. 2010; 31(4): 405-8.

13. World Medical Organization. Declaration of Helsinki. BMJ. 1996;313:1448-9.

14. Brasil. Ministério da Saúde. Resolução 196/96. O Plenário do Conselho Nacional de Saúde resolve aprovar diretrizes e normas regulamentadoras de pesquisas envolvendo seres humanos. Em 10 de outubro de 1996. 
15. Ciconelli RM, Ferraz MB, Santos W, Meinão I, Quaresma MR. Brazilian Portuguese version of SF-36. A reliable and valid quality of life outcome measure. Rev Bras Reumatol. 1999;39(3):143-50.

16. Crosby RD, Kolotkin RL, Williams GR. Defining clinically meaningful change in health-related quality of life. J Clin Epidemiol. 2003;56(5):395-407.

17. Hays RD, Farivar SS, Liu H. Approaches and recommendations for estimating minimally important differences for health-related quality of life measures. COPD. 2005;2(1):63-7.

18. Cohen J. Statistical power analysis in the behavioral sciences. 2nd ed. Hillsdale, NJ: Lawrence Erlbaum Associates, Inc; 1988.

19. Hagell P \& Westergren A. The significance of importance: an evaluation of ferrans and Powers' quality of life index. Quality of life research 2006; 15:867-76.

20. De Boer AG, Van Lanschot JJ, Stalmeier PF, et al. Is a single-item visual analogue scale as valid, reliable and responsive as multi-item scales in measuring quality of life?. Quality of Life Research 2004; 13(2):311-20.
21. Yousefian A, Leighton A, Fox K, Hartley D. Understanding the rural food environment perspectives of low-income parents. Rural Remote Health 2011;11(2):1631.

22. Márquez-Montero G, Loret de Mola C, BernabéOrtiz A, Smeeth L, Gilman RH, Miranda JJ. Healthrelated quality of life among urban and rural to urban migrant populations in Lima, Peru. Rev Peru Med Exp Salud Publica. 2011;28(1):35-41.

23. Tsai SY, Chi LY, Lee LS, Chou P. Health-related quality of life among urban, rural, and island community elderly in Taiwan. J Formos Med Assoc. 2004;103(3):196-204.

24. Pereira RF, et al. Contribuição dos domínios físico, social, psicológico e ambiental para a qualidade de vida global de idosos. Revista de Psiquiatria 2006; 28(1): 27-38

25. Sequeira A, Silva MN. O bem estar da pessoa idosa no meio rural. Análise Psicológica 2002; 20(3): 505-16.

26. Alencar NA, Aragão JCB, Ferreira MA, Dantas EHM. Avaliação da qualidade de vida em idosas residentes em ambientes urbano e rural. Revista brasileira de geriatria e gerontologia. 2010;13(1):103-9. 